\title{
Cooperative Localisation of a GPS-Denied UAV using Direction-of-Arrival Measurements
}

\author{
James S. Russell, Mengbin Ye, Brian D.O. Anderson, Hatem Hmam, Peter Sarunic
}

\begin{abstract}
A GPS-denied UAV (Agent B) is localised through 2 INS alignment with the aid of a nearby GPS-equipped UAV 3 (Agent A), which broadcasts its position at several time instants. 4 Agent B measures the signals' direction of arrival with respect to 5 Agent B's inertial navigation frame. Semidefinite programming 6 and the Orthogonal Procrustes algorithm are employed, and 7 accuracy is improved through maximum likelihood estimation. 8 The method is validated using flight data and simulations. A 9 three-agent extension is explored.
\end{abstract}

10 Index Terms-Localisation, INS alignment, Direction-of${ }_{11}$ Arrival Measurement, GPS-Denied, Semidefinite Programming 12

\section{INTRODUCTION}

14 Unmanned aerial vehicles (UAVs) play a central role in 15 many defence reconnaissance and surveillance operations. For16 mations of UAVs can provide greater reliability and coverage 17 when compared to a single UAV. To provide meaningful data 18 in such operations, all UAVs in a formation must have a ${ }_{19}$ common reference frame (typically the global frame). Tra20 ditionally, UAVs have access to the global frame via GPS. ${ }_{21}$ However, GPS signals may be lost in urban environments 22 and enemy controlled airspace (jamming). Overcoming loss 23 of GPS signal is a hot topic in research [1], and offers a range 24 of different problems in literature [2], [3].

25 Without access to global coordinates, a UAV must rely on its ${ }_{26}$ inertial navigation system (INS). Stochastic error in on-board ${ }_{27}$ sensor measurements causes the INS frame to accumulate drift. ${ }_{28}$ At any given time, drift can be characterised by a rotation and ${ }_{29}$ translation with respect to the global frame, and is assumed to so be independent between UAVs in a formation. INS frame drift ${ }_{31}$ therefore cannot be modelled deterministically. Information 32 from global and INS frames must be collected in order to ${ }_{3}$ determine the drift between frames and align the INS frame 34 with the global frame. We describe this process as cooperative 35 localisation when multiple vehicles interact for this purpose.

36 Signals of opportunity (SOP) such as AM/FM radio, digital 37 television or cellular communication can serve as references to 38 assist in characterizing the misalignment between navigation

The work of Russell, Ye, and Anderson was supported by the Australian Research Council (ARC) Discovery Project DP-160104500, by 111-Project No. D17019, and by Data61-CSIRO. Ye was supported by an Australian Government Research Training Program (RTP) Scholarship.

J.S. Russell, M. Ye and B.D.O. Anderson are with the Research School of Engineering, Australian National University, Canberra, Australia \{u5542624, mengbin.ye, brian.anderson\}@anu.edu.au. B.D.O. Anderson is also with Hangzhou Dianzi University, Hangzhou, China, and with Data61-CSIRO in Canberra, Australia. H. Hmam and P. Sarunic are with Australian Defence Science and Technology Group (DST Group), Edinburgh, Australia \{hatem.hmam, peter.sarunic\}edst.defence.gov.au. frames of multiple agents. Recent contributions in this field 39 include [4]-[6]. In contexts where SOP are either unavailable 40 or unreliable, various measurement types such as distance 41 between agents and direction of arrival of a signal (we 42 henceforth call $\mathrm{DOA}^{1}$ ) can be used for this process. In the ${ }_{43}$ context of UAVs, additional sensors add weight and consume ${ }_{44}$ power. As a result, one generally aims to minimise the number 45 of measurement types required for localisation. This paper 46 studies a cooperative approach to localisation using DOA 47 measurements.

When two or more GPS-enabled UAVs can simultaneously 49 measure directions with respect to the global frame towards 50 the GPS-denied UAV, the location of the GPS-denied UAV is 51 given by the point minimising distances to the half-line loci de- 52 rived from the directional measurements [7]-[9]. Operational 53 requirements may limit the number of nearby GPS-enabled 54 UAVs to one single agent. We therefore seek a solution which 55 does not require simultaneous measurements to a single point. 56

When the GPS-denied agent is able to simultaneously mea- 57 sure directions with respect to its local INS frame towards mul- 58 tiple landmarks with known global coordinates, triangulation- 59 based measurements can be used to achieve localisation. This 60 problem is studied in three-dimensional space in [10], and 61 in two-dimensional space in [11], [12]. If only one landmark 62 bearing can be measured at any given time, a bearing-only 63 SLAM algorithm may be used to progressively construct a 64 map of the environment on the condition each landmark is 65 seen at least twice. Alignment of a GPS-denied agent's INS 66 frame could then be achieved by determining the rotation 67 and translation between the map's coordinate frame and the 68 global coordinate frame. In practice, landmark locations may 69 be unknown, or there may be no guarantee they are stationary 70 or permanent, and hence we require a localisation algorithm 71 which is independent of landmarks in the environment. Iter- 72 ative filtering methods such as the Extended Kalman Filter ${ }_{73}$ (EKF) are often required when drift is significant between 74 updates. In [13], an EKF is used to estimate drift in the 75 context of marine localisation. In our problem context the drift 76 is sufficiently slow to be modelled as stationary over short 77 periods. We are motivated to formulate a localisation algorithm 78 which does not involve an iterative filtering technique. $\quad 79$

Without reliance on landmarks, the only directional mea- 80 surements available are between the GPS-denied and the 81 GPS-enabled UAVs. Given the small size of their airframes 82 with respect to their separation distance, these UAVs are ${ }_{83}$

\footnotetext{
${ }^{1}$ A bearing generally describes a scalar measurement between two points in a plane, whereas a direction-of-arrival is a vector measurement between two points in three-dimensional ambient space (as considered in this paper).
} 
84 modelled as point agents, and therefore one single directional 85 measurement is available at any given time. A stationary target 86 is localised by an agent using bearing-only measurements in 87 two-dimensional space [14], [15], and in three-dimensional 88 space [16]. A similar problem is considered in [17], in which 89 a mobile source is localised using measurements received at a 90 stationary receiver using an iterative filtering technique. How${ }_{91}$ ever, for operational reasons, the agent requiring localisation 92 may be unable to broadcast signals, or agents involved may 93 not be allowed to remain stationary. In such instances, the 94 approaches in [14]-[17] are not suitable. Commonly used 95 computer vision techniques such as structure from motion 96 [18] require directional measurements towards multiple sta97 tionary points or towards a stationary point from multiple 98 known positions. This is not possible in our problem context. 99 The measurement and motion requirements we are imposing 100 therefore represent a significant technical challenge. One al101 gorithm satisfying all the requirements above was proposed 102 in [19], in which two agents perform sinusoidal motion in 103 two-dimensional ambient space. Directional measurements are 104 used to obtain the distance between Agents A and B, but 105 localisation of B in the global frame is not achieved.

106 Motivated by interest from Australia's Defence Science and 107 Technology Group, this paper seeks to address the problem of 108 localising a GPS-denied UAV with the assistance of a GPS109 enabled UAV, which we will call Agent B and A respec110 tively. Both agents move arbitrarily in three-dimensional space. 111 Agent B navigates using an INS frame. Agent A broadcasts 112 its position in the global coordinate frame at discrete instants ${ }_{113}$ in time. For each broadcast, Agent B is able to take a DOA 114 measurement towards Agent A.

115 The problem setup and the solution we propose are both 116 novel. In particular, while the literature discussed above con117 siders certain aspects from the following list, none consider 118 all of the following aspects simultaneously:

119 - The network consists of only two mobile agents (and 120 is therefore different to the sensor network localisation 121 problems in the literature).

122 - There is no a priori knowledge or sensing of a stationary 123 reference point in the global frame.

124 - Both UAVs are free to execute arbitrary motion in three125 dimensional space, with the exception of a small number 126 of geometrically unsolvable trajectory pairs ${ }^{2}$.

127 - Cooperation occurs through unidirectional signal trans128 mission. Agent A broadcasts its global position (acquired 129 using GPS) to Agent B (which is GPS-denied).

130 When performing non-routine operations in unfamiliar en131 vironments, any combination of these four aspects may be 132 required with short notice. As a result, we are motivated 133 to determine a reliable general solution to the cooperative 134 localisation problem.

135 In [20], this problem is studied in two-dimensional space ${ }_{136}$ using bearing measurements, but the added (third) dimension ${ }_{137}$ in our paper means 2 scalar quantities, not 1 , are obtained per

\footnotetext{
${ }^{2}$ No constraints exist on the trajectories other than the physical limitations of the aircraft. See Section VI-C for further details on unsuitable trajectories.
}

measurement. This significantly complicates the problem, thus 138 requiring new techniques to be introduced.

In our proposed solution, we localise Agent B by identifying 140 the relationship between the global frame (navigated by Agent 141 A) and the inertial navigation frame of Agent B. The rela- 142 tionship is identified by solving a system of linear equations 143 for a set of unknown variables. The nature of the problem ${ }_{144}$ means quadratic constraints exist on some of the variables. To 145 improve robustness against noisy measurements, we exploit ${ }_{146}$ the quadratic constraints and use semidefinite programming ${ }_{147}$ (SDP) and the Orthogonal Procrustes algorithm to obtain an 148 initial solution for maximum likelihood (ML) estimation; this 149 combined approach is a key novel contribution of this paper. 150

We evaluate the performance of the algorithm by (i) using a 151 real set of trajectories and (ii) using Monte Carlo simulations. 152 Sets of unsuitable trajectories are identified, in which our 153 proposed method cannot feasibly obtain a unique solution. 154 Finally, we explore an extension of the algorithm to a three- 155 agent network in which two agents are GPS-denied. 156

The rest of the paper is structured as follows. In Section II 157 the problem is formalised. In Section III a localisation method 158 using a linear equation formulation is proposed. Section IV ${ }_{159}$ extends this method to semidefinite programming to produce 160 a more robust localisation algorithm. In Section V, a maximum 161 likelihood estimation method is presented to refine results 162 further. Section VI presents simulation results to evaluate the ${ }_{163}$ performance of the combined localisation algorithm. Section 164 VII extends the localisation algorithm to a three-agent net- 165 work. The paper is concluded in Section VIII. ${ }^{3}$

\section{Problem Definition}

167

Two agents, which we call Agent A and Agent B, travel 168 along arbitrary trajectories in three-dimensional space. Agent ${ }_{169}$ A has GPS and therefore navigates with respect to the global 170 frame. Because Agent B cannot access GPS, it has no ability 171 to self-localise in the global frame, but can self-localise and ${ }_{172}$ navigate in a local inertial frame by integrating gyroscope and 173 accelerometer measurements.

This two-agent localisation problem involves 4 frames as 175 in Figure 1. The importance of each frame, and its use in 176 obtaining the localisation, will be made clear in the sequel. 177 Frames are labelled as follows:

- The global frame is $A_{1}$ (available only to Agent $\mathrm{A}$ ),

- the body-centred INS frame of Agent B (axes of $B_{2}$ and $B_{3}$ are parallel by definition)is denoted $B_{3}$,

- the body-fixed frame of Agent B is denoted $B_{4}$.

The expression of directional measurements with respect 184 to the INS frame in vector form motivates the definition of 185 the body-centred frame $B_{3}$. Later, we find that differences in 186

\footnotetext{
${ }^{3}$ Early sections in this paper (covering up to and including employment of Orthogonal Procrustes algorithm) appeared in less detail in the conference paper [21]. Additions have been made to these sections - the literature review is now more extensive, and the role of different coordinate frames is much more explicitly set out; the algorithm's performance is now validated on real flight trajectories. Analysis of unsuitable trajectories, ML refinement and the three-agent extension are further extensions beyond [21].
} 
187 body-fixed frame azimuth and elevation measurement noise 188 motivate the use of $B_{4}$ for maximum likelihood estimation.

189 Note that agents $\mathrm{A}$ and $\mathrm{B}$ are denoted by a single letter, 190 whereas frames $A_{1}$ and $B_{i}$ for $i=2,3,4$ are denoted by a 191 letter-number pair. Let $\boldsymbol{p}_{\boldsymbol{J}}^{\boldsymbol{I}_{\mathbf{O}}}(k)$ denote the position of Agent $J$ 192 in coordinates of frame $I_{0}$ at the $k^{t h}$ time instant. Let $u_{J}, v_{J}$, $193 w_{J}$ denote Agent J's coordinates in the global frame $\left(A_{1}\right)$, and $194 x_{J}, y_{J}, z_{J}$ denote Agent J's coordinates in Agent B's local 195 INS frame $\left(B_{2}\right)$. It follows that:

196

197

$$
\begin{gathered}
\boldsymbol{p}_{\boldsymbol{A}}^{\boldsymbol{A}_{\mathbf{1}}}(k)=\left[u_{A}(k), v_{A}(k), w_{A}(k)\right]^{\top} \\
\boldsymbol{p}_{\boldsymbol{B}}^{\boldsymbol{B}_{\mathbf{2}}}(k)=\left[x_{B}(k), y_{B}(k), z_{B}(k)\right]^{\top}
\end{gathered}
$$

199 The rotation and translation of Agent B's local INS frame $200\left(B_{2}\right)$ with respect to the global frame $\left(A_{1}\right)$ evolves via drift. 201 Although this drift is significant over long periods, frame $B_{2}$ 202 can be modelled as stationary with respect to frame $A_{1}$ over 203 short intervals ${ }^{4}$. During these short intervals, the following 204 measurement process occurs multiple times. At each time 205 instant $k$, the following four activities occur simultaneously 206 : positive if the $z$ component of the unit vector towards 226 Agent A is positive.

227

The problem addressed in this paper, namely the localisation 228 of Agent B, is achieved if we can determine the relationship 229 between the global frame $A_{1}$ and the local INS frame $B_{2} .{ }_{230}$ This information can be used to determine global coordinates 231 of Agent $\mathrm{B}$ at each time instant $k$ :

$$
\boldsymbol{p}_{\boldsymbol{B}}^{\boldsymbol{A}_{1}}(k)=\left[u_{B}(k), v_{B}(k), w_{B}(k)\right]^{\top}
$$

Passing between the global frame $\left(A_{1}\right)$ and the local INS 234 frame of Agent B $\left(B_{2}\right)$ is achieved by a rotation of frame axes 235 (defined by a rotation matrix, call it $\boldsymbol{R}_{\boldsymbol{A}_{1}}^{\boldsymbol{B}_{2}}$ ) and translation $\boldsymbol{t}_{\boldsymbol{A}_{1}{ }_{236}}$ of frame. For instance, the coordinate vector of the position 237 of Agent A referenced to the INS frame of Agent B is: 238

$$
\boldsymbol{p}_{\boldsymbol{A}}^{\boldsymbol{B}_{2}}(k)=\boldsymbol{R}_{\boldsymbol{A}_{1}}^{\boldsymbol{B}_{2}} \boldsymbol{p}_{\boldsymbol{A}}^{\boldsymbol{A}_{1}}(k)+\boldsymbol{t}_{\boldsymbol{A}_{1}}^{\boldsymbol{B}_{2}}
$$

We therefore have

$$
\boldsymbol{p}_{\boldsymbol{B}}^{\boldsymbol{A}_{1}}(k)=\boldsymbol{R}_{\boldsymbol{A}_{1}}^{\boldsymbol{B}_{2} \top}\left(\boldsymbol{p}_{\boldsymbol{B}}^{\boldsymbol{B}_{2}}(k)-\boldsymbol{t}_{\boldsymbol{A}_{1}}^{\boldsymbol{B}_{\mathbf{2}}}\right)
$$

where $\boldsymbol{R}_{A_{1}}^{B_{2} \top}=R_{B_{2}}^{A_{1}}$ and $-\boldsymbol{R}_{A_{1}}^{B_{2} \top} \boldsymbol{t}_{\boldsymbol{A}_{1}}^{\boldsymbol{B}_{2}}=\boldsymbol{t}_{\boldsymbol{B}_{2}}^{\boldsymbol{A}_{2}}$. The locali- 242 sation problem can be reduced to solving for $\boldsymbol{R}_{\boldsymbol{A}_{1}}^{B_{2}} \in S O(3){ }_{243}$ with entries $r_{i j}$ and $\boldsymbol{t}_{\boldsymbol{A}_{1}}^{\boldsymbol{B}_{\mathbf{2}}} \in \mathbb{R}^{3}$ with entries $t_{i}$.

The matrix $\boldsymbol{R}_{\boldsymbol{A}_{1}}^{\boldsymbol{B}_{2}}$ is a rotation matrix if and only if 245 $\boldsymbol{R}_{\boldsymbol{A}_{1}}^{\boldsymbol{B}_{2}} \boldsymbol{R}_{\boldsymbol{A}_{1}}^{\boldsymbol{B}_{2} \top}=I_{3}$ and $\operatorname{det}\left(\boldsymbol{R}_{\boldsymbol{A}_{1}}^{\boldsymbol{B}_{2}}\right)=1$. As will be seen in the 246 sequel, these constraints are equivalent to a set of quadratic 247 constraints on the entries of $\boldsymbol{R}_{\boldsymbol{A}_{1}}^{\boldsymbol{B}_{\mathbf{1}}}$. In total there are 12 entries 248 of $\boldsymbol{R}_{\boldsymbol{A}_{1}}^{\boldsymbol{B}_{2}}$ and $\boldsymbol{t}_{\boldsymbol{A}_{1}}^{\boldsymbol{B}_{2}}$ to be found as we work directly with $r_{i j}$. ${ }_{249}$

\section{LineAR SyStem METHOD}

250

This section presents a linear system (LS) method to solving 251 the localisation problem. Given enough measurements, the 252 linear system approach can achieve exact localisation when 253 using noiseless DOA measurements, so long as Agents A and 254 $B$ avoid a set of unsuitable trajectories (which are detailed 255 in Section VI-C) in which rank-deficiency is encountered. 256 Building on this, Section IV introduces non-linear constraints 257 to the linear problem defined in this section to improve 258 accuracy in the presence of noise.

\section{A. Forming a system of linear equations}

260

The following analysis holds for all $k$ instants in time, 261 hence we drop the argument $k$. The DOA measurement can be 262 represented by a unit vector pointing from Agent B to Agent 263 A. This vector is defined by azimuth and elevation angles $\theta{ }_{264}$ and $\phi$ referenced to the local INS frame $B_{2}$, and its coordinates 265 in the frame $B_{2}$ are given by:

266

$$
\hat{\boldsymbol{q}}(\theta, \phi)=\left[\hat{q_{1}}, \hat{q_{2}}, \hat{q_{3}}\right]=[\cos \theta \cos \phi, \sin \theta \cos \phi, \sin \phi]^{\top} \quad \text { (6) }{ }_{267}
$$

Define $\bar{q} \doteq\left\|\boldsymbol{p}_{\boldsymbol{A}}^{\boldsymbol{B}_{\mathbf{2}}}-\boldsymbol{p}_{\boldsymbol{B}}^{\boldsymbol{B}_{\mathbf{2}}}\right\|$ as the Euclidean distance between 268 Agent A and Agent B (which is not available to either agent). 269 Scaling to obtain the unit vector $\hat{\boldsymbol{q}}$ gives

$$
\hat{\boldsymbol{q}}(\theta, \phi)=\frac{1}{\bar{q}}\left[x_{A}-x_{B}, \quad y_{A}-y_{B}, \quad z_{A}-z_{B}\right]^{\top}
$$

Fig. 1. Illustration of coordinate frames in a two-dimensional space 
272 Applying equation (4) yields:

$273 \quad\left[\begin{array}{l}\hat{q_{1}} \\ \hat{q_{2}} \\ \hat{q_{3}}\end{array}\right]=\frac{1}{\bar{q}}\left[\begin{array}{l}r_{11} u_{A}+r_{12} v_{A}+r_{13} w_{A}+t_{1}-x_{B} \\ r_{21} u_{A}+r_{22} v_{A}+r_{23} w_{A}+t_{2}-y_{B} \\ r_{31} u_{A}+r_{32} v_{A}+r_{33} w_{A}+t_{3}-z_{B}\end{array}\right]$

274 The left hand vector is calculated directly from DOA mea275 surements. Cross-multiplying entries 1 and 3 of both vectors 276 eliminates the unknown $\bar{q}$, and rearranging yields:

$$
\begin{array}{ll} 
& \left(u_{A} \hat{q_{3}}\right) r_{11}+\left(v_{A} \hat{q_{3}}\right) r_{12}+\left(w_{A} \hat{q_{3}}\right) r_{13}-\left(u_{A} \hat{q_{1}}\right) r_{31} \\
{ }_{277} & -\left(v_{A} \hat{q_{1}}\right) r_{32}-\left(w_{A} \hat{q_{1}}\right) r_{33}+\left(\hat{q_{3}}\right) t_{1}-\left(\hat{q_{1}}\right) t_{3} \\
& =\left(\hat{q_{3}}\right) x_{B}-\left(\hat{q_{1}}\right) z_{B}
\end{array}
$$

279 Similarly, from the second and third entries in (8)

$$
\begin{array}{ll} 
& \left(u_{A} \hat{q_{3}}\right) r_{21}+\left(v_{A} \hat{q_{3}}\right) r_{22}+\left(w_{A} \hat{q_{3}}\right) r_{23}-\left(u_{A} \hat{q_{2}}\right) r_{31} \\
280 & -\left(v_{A} \hat{q_{2}}\right) r_{32}-\left(w_{A} \hat{q_{2}}\right) r_{33}+\left(\hat{q_{3}}\right) t_{2}-\left(\hat{q_{2}}\right) t_{3} \\
& =\left(\hat{q_{3}}\right) y_{B}-\left(\hat{q_{2}}\right) z_{B}
\end{array}
$$

282 Notice that both equations (9) and (10) are linear in the 283 unknown $r_{i j}$ and $t_{i}$ terms. Given a series of $K$ DOA mea284 surements (each giving $\phi(k), \theta(k)$ ), (9) and (10) can then be 285 used to construct the following system of linear equations:

$$
\boldsymbol{A} \boldsymbol{\Psi}=\boldsymbol{b}, \quad \boldsymbol{A} \in \mathbb{R}^{2 K \times 12}
$$

${ }_{287}$ where $\boldsymbol{A}, \boldsymbol{b}$ are completely known, containing $\theta(k), \phi(k), \boldsymbol{p}_{\boldsymbol{A}}^{\boldsymbol{A}_{1}}$ ${ }_{288}$ and $\boldsymbol{p}_{\boldsymbol{B}}^{\boldsymbol{B}_{\mathbf{2}}}$. The 12-vector of unknowns $\boldsymbol{\Psi}$ is defined as:

${ }_{289} \boldsymbol{\Psi}=\left[\begin{array}{llllllllll}r_{11} & r_{12} & r_{13} & \ldots & r_{31} & r_{32} & r_{33} & t_{1} & t_{2} & t_{3}\end{array}\right]^{\top}$

290 Entry-wise definitions of $\boldsymbol{A}$ and $\boldsymbol{b}$ are provided in an extended 291 version of this paper [22]. These entries of $\Psi$ can be used to 292 reconstruct the trajectory of Agent B in the global frame using 293 (5), and therefore solving (11) for $\Psi$ constitutes as a solution 294 to the localisation problem. In the noiseless case, if $K \geq 6$ 295 and $\boldsymbol{A}$ is of full column rank, equation (11) will be solvable.

\section{${ }_{296}$ B. Example of LS method in noiseless case}

297 We demonstrate the linear system method using trajectories 298 performed by aircraft operated by the Australian Defence 299 Science and Technology Group. Positions of both Agent A 300 and B within the global frame and Agent B within the INS 301 frame were measured by on-board instruments, whereas we 302 generated a set of calculated DOA values using the above 303 recorded real measurements.

304 These trajectories are plotted in Figure 2. We will make 305 additional use of this trajectory pair in the noisy measurement 306 case presented in Section IV, and in the maximum likelihood 307 estimation refinement of the noisy case localisation result in 308 Section V. Extensive Monte Carlo simulations demonstrating 309 localisation for a large number of realistic ${ }^{5}$ flight trajectories 310 are left to the noisy measurement case.

${ }_{311}$ The quantities $\boldsymbol{R}_{\boldsymbol{A}_{1}}^{\boldsymbol{B}_{2}}$ and $\boldsymbol{t}_{\boldsymbol{A}_{1}}^{\boldsymbol{B}_{2}}$, and the DOA measurements 312 are tabulated in the extended version of this paper [22]. Using ${ }_{313}$ (11), $\boldsymbol{R}_{\boldsymbol{A}_{1}}^{\boldsymbol{B}_{2}}$ and $\boldsymbol{t}_{\boldsymbol{A}_{1}}^{\boldsymbol{B}_{2}}$ were obtained exactly for the given flight 314 trajectories; the solution is the green line in Fig. 2.

\footnotetext{
${ }^{5}$ By realistic, we mean that the distance separation between successive measurements is consistent with UAV flight speeds and ensures the UAV does not exceed an upper bound on the turn/climb rate. Further detail is provided in the extended version of this paper [22]
}

This section presents a semidefinite programming (SDP) 316 method for localisation, extending from the linear system (LS) 317 approach presented in Section III. This method reduces the 318 minimum required number of DOA measurements to obtain a ${ }_{319}$ unique solution, and is more robust than LS in terms of DOA 320 measurement noise and unsuitable trajectories are reduced. 321 Results from this section will serve as an initialisation of our ${ }_{322}$ localisation method, which will be optimised using maximum 323 likelihood estimation in Section V.

324

Rank-relaxed SDP is used to incorporate the quadratic con- 325 straints on certain entries of $\boldsymbol{\Psi}$ arising from the properties of ${ }_{326}$ rotation matrices. The inclusion of rotation matrix constraints 327 in SDP problems has been used previously to jointly estimate ${ }_{328}$ the attitude and spin-rate of a satellite [23], and in camera pose ${ }_{329}$ estimation using SFM techniques when directional measure- 330 ments are made to multiple points simultaneously [24]. We ${ }_{331}$ now apply this technique in a novel context to achieve INS 332 alignment of Agent B, sufficient for its localisation. Finally, 333 the Orthogonal Procrustes algorithm (O) is used to compensate ${ }_{334}$ for the rank relaxation of the SDP.

335

\section{A. Quadratic constraints on entries of $\Psi$}

336

Rank-relaxed semidefinite programming (in the presence 337 of inexact or noise contaminated data) benefits from the ${ }_{338}$ inclusion of quadratic constraint equations. We now identify 339 21 quadratic and linearly independent constraint equations on 340 entries of $\boldsymbol{R}_{\boldsymbol{A}_{1}}^{\boldsymbol{B}_{2}}$, which all appear in $\boldsymbol{\Psi}$ in (12). Recall the 341 orthogonality property of rotation matrices; by computing each 342 entry of $\boldsymbol{R}_{\boldsymbol{A}_{\mathbf{1}}}^{\boldsymbol{B}_{\mathbf{2}}} \boldsymbol{R}_{\boldsymbol{A}_{1}}^{\boldsymbol{B}_{\mathbf{2}}{ }^{\top}}$ and setting these equal to entries of $\boldsymbol{I}_{\mathbf{3}}, 343$ and denoting the $i^{\text {th }}$ entry of $\boldsymbol{\Psi}$ as $\psi_{i}$, we define constraints: ${ }_{344}$

$$
\begin{array}{ll}
C_{i}=\psi_{3 i-2}^{2}+\psi_{3 i-1}^{2}+\psi_{3 i}^{2}-1=0, \quad i=1,2,3 \\
C_{4}=\psi_{1} \psi_{4}+\psi_{2} \psi_{5}+\psi_{3} \psi_{6}=0 \\
C_{5}=\psi_{1} \psi_{7}+\psi_{2} \psi_{8}+\psi_{3} \psi_{9}=0 \\
C_{6}=\psi_{4} \psi_{7}+\psi_{5} \psi_{8}+\psi_{6} \psi_{9}=0
\end{array}
$$

To simplify notation we call $C_{j: k}$ the set of constraints $C_{i}$ for 350 $i=j, . ., k$. Similarly, by computing each entry of $\boldsymbol{R}_{\boldsymbol{A}_{1}}^{\boldsymbol{B}_{\mathbf{2}}^{\top}} \boldsymbol{R}_{\boldsymbol{A}_{1}{ }^{351}}^{\boldsymbol{B}_{2}}$ and setting these equal to $\boldsymbol{I}_{\mathbf{3}}$, we define constraints $C_{7: 12}, 352$ which are omitted due to space limitations, and notice that ${ }_{353}$ the sets $C_{1: 6}$ and $C_{7: 12}$ are clearly equivalent. 354

Further constraints are required to ensure $\operatorname{det}\left(\boldsymbol{R}_{\boldsymbol{A}_{1}}^{\boldsymbol{B}_{\mathbf{2}}}\right)=1.355$ Cramer's formula states that $\boldsymbol{R}_{\boldsymbol{A}_{1}}^{\boldsymbol{B}_{2}{ }^{-1}}=\operatorname{adj}\left(\boldsymbol{R}_{\boldsymbol{A}_{1}}^{\boldsymbol{B}_{2}}\right) / \operatorname{det}\left(\boldsymbol{R}_{\boldsymbol{A}_{1}}^{\boldsymbol{B}_{2}}\right), 356$ where $\operatorname{adj}\left(\boldsymbol{R}_{\boldsymbol{A}_{1}}^{\boldsymbol{B}_{2}}\right)$ denotes the adjugate matrix of $\boldsymbol{R}_{\boldsymbol{A}_{\mathbf{1}}}^{\boldsymbol{B}_{\mathbf{2}}}$. Orthog- 357 onality of $\boldsymbol{R}_{\boldsymbol{A}_{1}}^{\boldsymbol{B}_{2}}$ implies $\boldsymbol{R}_{\boldsymbol{A}_{1}}^{\boldsymbol{B}_{2}}=\operatorname{adj}\left(\boldsymbol{R}_{\boldsymbol{A}_{1}}^{\boldsymbol{B}_{2}}\right)^{\top}$. By computing 358 entries of the first column of $\boldsymbol{Z}=\boldsymbol{R}_{\boldsymbol{A}_{1}}^{\boldsymbol{B}_{\mathbf{1}}}-\operatorname{adj}\left(\boldsymbol{R}_{\boldsymbol{A}_{1}}^{\boldsymbol{B}_{2}}\right)^{\top}$ and 359 setting these equal to 0 , we define constraints $C_{13: 15}$ :

360

$$
\begin{aligned}
& C_{13}=\psi_{1}-\left(\psi_{5} \psi_{9}-\psi_{6} \psi_{8}\right)=0 \\
& C_{14}=\psi_{4}-\left(\psi_{3} \psi_{8}-\psi_{2} \psi_{9}\right)=0 \\
& C_{15}=\psi_{7}-\left(\psi_{2} \psi_{6}-\psi_{3} \psi_{5}\right)=0
\end{aligned}
$$$$
\text { (14b) } 362
$$

Similarly, by computing the entries of the second and third 365 columns of $\boldsymbol{Z}$ and setting these equal to 0 , we define con- 366 straints $C_{16: 18}$ and $C_{19: 21}$ respectively. Due to space limita- 367 tions, we omit presenting them. The complete set $C_{1: 21} \doteq C_{\Psi}{ }_{368}$ 
369 constrains $\boldsymbol{R}_{\boldsymbol{A}_{1}}^{\boldsymbol{B}_{2}}$ to be a rotation matrix. The set of constraints 370 is not an independent set, e.g. $\boldsymbol{C}_{\mathbf{1 : 6}}$ is equivalent to $\boldsymbol{C}_{\mathbf{7 : 1 2}}$. The 371 benefits of the inclusion of dependent constraints is discussed 372 further in Section IV-C.

373 Due to these additional relations, localisation requires az374 imuth and elevation measurements at a minimum of 4 instants 375 only $(K=4)$, as opposed to 6 instants required in Section III.

\section{B. Formulation of the Semidefinite Program}

377 The goal of the semidefinite program is to obtain:

378

$$
\underset{\boldsymbol{\Psi}}{\operatorname{argmin}}\|\boldsymbol{A} \boldsymbol{\Psi}-\boldsymbol{b}\|
$$

379 subject to $C_{\Psi}$. Equivalently, we seek $\operatorname{argmin}_{\Psi}\|\boldsymbol{A} \boldsymbol{\Psi}-\boldsymbol{b}\|^{2}$ 380 subject to $C_{\Psi}$. We define the inner product of two matrices $\boldsymbol{U}$ 381 and $\boldsymbol{V}$ as $\langle\boldsymbol{U}, \boldsymbol{V}\rangle=\operatorname{trace}\left(\boldsymbol{U} \boldsymbol{V}^{\top}\right)$. One obtains

$$
\|\boldsymbol{A} \boldsymbol{\Psi}-\boldsymbol{b}\|^{2}=\langle\boldsymbol{P}, \boldsymbol{X}\rangle
$$

384 where $\boldsymbol{P}=\left[\begin{array}{ll}\boldsymbol{A} & \boldsymbol{b}\end{array}\right]^{\top}\left[\begin{array}{ll}\boldsymbol{A} & \boldsymbol{b}\end{array}\right]$ and $\boldsymbol{X}=\left[\boldsymbol{\Psi}^{\top}-1\right]^{\top}\left[\boldsymbol{\Psi}^{\top}-1\right]$ 385 and $\boldsymbol{X}$ is a rank 1 positive-semidefinite matrix ${ }^{6}$. The con386 straints $C_{\Psi}$ can also be expressed in inner product form. For ${ }_{387} i=1, \ldots, 21, C_{i}=0$ is equivalent to $\left\langle\boldsymbol{Q}_{\boldsymbol{i}}, \boldsymbol{X}\right\rangle=0$ for 38 some easily determined $\boldsymbol{Q}_{\boldsymbol{i}}$. Solving for $\boldsymbol{\Psi}$ in (15) is therefore 389 equivalent to solving for:

$\begin{array}{rlr}390 & \underset{\Psi}{\operatorname{argmin}}\langle\boldsymbol{P}, \boldsymbol{X}\rangle \\ 391 & \boldsymbol{X} \geq 0 \\ 392 & \operatorname{rank}(\boldsymbol{X})=1 \\ 393 & \boldsymbol{X}_{\mathbf{1 3}, \mathbf{1 3}} & =1 \\ & & \\ 394 & \left\langle\boldsymbol{Q}_{\boldsymbol{i}}, \boldsymbol{X}\right\rangle & =0 \quad i=1, \ldots, 21\end{array}$

\section{${ }_{396}$ C. Rank Relaxation of Semidefinite Program}

397 This semidefinite program is a reformulation of a quadrati398 cally constrained quadratic program (QCQP). Computationally 399 speaking, QCQP problems are generally NP-hard. A close 400 approximation to the true solution can be obtained in polyno401 mial time if the rank 1 constraint on $\boldsymbol{X}$, i.e. (22), is relaxed. 402 A full explanation of semidefinite relaxation, and discussion 403 on its applicability can be found in [25]. This relaxation 404 significantly increases the dimension of the SDP solver's co405 domain. A notable consequence is that dependent constraints 406 which are linearly independent over $\mathbb{R}$ within $\boldsymbol{C}_{\boldsymbol{\Psi}}$, such as sets ${ }_{407} C_{1: 6}$ and $\boldsymbol{C}_{7: 12}$, cease to be redundant when expressed as in 408 (21). Hypothesis testing using extensive simulations confirmed 409 with confidence above $95 \%$ that inclusion of quadratically 410 dependent constraints improves the localisation accuracy.

${ }_{411}$ The solution $\boldsymbol{X}$ obtained through rank-relaxed SDP is 412 typically not a rank 1 matrix when DOA measurements are 413 noisy. However the largest singular value of $\boldsymbol{X}$ is generally ${ }_{414}$ multiple orders of magnitude greater than the second largest 415 singular value. A rank 1 approximation to $\boldsymbol{X}$, which we call ${ }_{416} \hat{\boldsymbol{X}}$, is obtained by evaluating the singular value decomposition 417 of $\boldsymbol{X}$, then setting all singular values except the largest equal

\footnotetext{
${ }^{6}$ All matrices $\boldsymbol{M}$ which can be expressed in the form of $\boldsymbol{M}=\boldsymbol{v}^{\top} \boldsymbol{v}$ where $\boldsymbol{v}$ is a row vector are positive-semidefinite matrices.
}

to zero. From $\hat{\boldsymbol{X}}$, one can then use the definition of $\boldsymbol{X}{ }_{418}$ to obtain the approximation of $\boldsymbol{\Psi}$, which we will call $\hat{\boldsymbol{\Psi}} .419$ Entries $\hat{\psi}_{i}$ for $i=10,11,12$ can be used immediately to ${ }_{420}$ construct an estimate for $\boldsymbol{t}_{\boldsymbol{A}_{1}}^{\boldsymbol{B}_{2}}$, which we will call $\overline{\boldsymbol{t}}$. Entries 421 $\hat{\psi}_{i}$ for $i=1, \ldots, 9$ will be used to construct an intermediate ${ }_{422}$ approximation of $\boldsymbol{R}_{\boldsymbol{A}_{1}}^{\boldsymbol{B}_{2}}$, which we call $\widehat{\boldsymbol{R}}$, and which we will ${ }_{423}$ refine further.

\section{Orthogonal Procrustes Algorithm}

425

Due to the relaxation of the rank constraint (19) on $\boldsymbol{X}$, it is 426 no longer guaranteed that entries of $\hat{\boldsymbol{\Psi}}$ strictly satisfy the set of ${ }_{427}$ constraints $C_{\Psi}$. Specifically, $\widehat{\boldsymbol{R}}$ may not be a rotation matrix. ${ }_{428}$ The Orthogonal Procrustes algorithm is a commonly used tool ${ }_{429}$ to determine the closest orthogonal matrix (denoted $\overline{\boldsymbol{R}}$ ) to a 430 given matrix, $\widehat{\boldsymbol{R}}$. This is given by $\overline{\boldsymbol{R}}=\operatorname{argmin}_{\boldsymbol{\Omega}}\|\boldsymbol{\Omega}-\widehat{\boldsymbol{R}}\|_{F}, 431$ subject to $\boldsymbol{\Omega} \boldsymbol{\Omega}^{\top}=I$, where \|\|$_{F}$ is the Frobenius norm. 432

When noise is high, the above method occasionally returns ${ }_{433}$ $\overline{\boldsymbol{R}}$ such that $\operatorname{det}(\overline{\boldsymbol{R}})=-1$. In this case, we employ a special 434 case of the Orthogonal Procrustes algorithm [26] to ensure we 435 obtain rotation matrices and avoid reflections by flipping the 436 last column in one of the unitary matrix factors of the singular 437 value decomposition.

The matrix $\overline{\boldsymbol{R}}$ and vector $\overline{\boldsymbol{t}}$ are the final estimates of $\boldsymbol{R}_{\boldsymbol{A}_{1}}^{\boldsymbol{B}_{2}}{ }_{439}$ and $\boldsymbol{t}_{\boldsymbol{A}_{1}}^{\boldsymbol{B}_{2}}$ using semidefinite programming and the Orthogonal 440 Procrustes algorithm. The estimate of Agent B's position in 441 the global frame is $\overline{\boldsymbol{p}_{B}^{\boldsymbol{A}_{1}}}=\overline{\boldsymbol{R}}^{\top}\left(\boldsymbol{p}_{B}^{B_{2}}-\overline{\boldsymbol{t}}\right)$.

For convenience, we use $\mathrm{SDP}+\mathrm{O}$ to refer to the process 443 of solving a rank-relaxed semidefinite program, and then 444 applying the Orthogonal Procrustes algorithm to the result. ${ }_{445}$

\section{E. Example of $S D P+O$ method with noisy DOA measurements 446}

In this subsection, we apply the $\mathrm{SDP}+\mathrm{O}$ method to perform 447 localisation in a noisy DOA measurement case using the real ${ }_{448}$ trajectory example from Section III. A popular practice for ${ }_{449}$ performing DOA measurements from Agent B towards Agent 450 $A$ is to use fixed RF-antennas and/or optical sensors on board 451 Agent B's airframe. The horizontal RF antenna typically has 452 a larger aperture (generally around 4 times, owing to the 453 physical layout of a fixed-wing UAV) than the vertical RF ${ }_{454}$ antenna. As a result, errors in azimuth and elevation measure- 455 ments, referenced to the body-fixed frame $B_{4}$, are modelled 456 by independent zero-mean Gaussian distributed variables with ${ }_{457}$ different standard deviations, denoted $\sigma_{\Theta}$ and $\sigma_{\Phi}$. 458

Strictly speaking, physical sensors return azimuth and ele- 459 vation measurements in the interval $\left[0^{\circ}, 360^{\circ}\right)$, which means 460 that each noise is expected to follow a von Mises distribution, 461 which generalises a Gaussian distribution to a circle [27]. 462 In our case, we approximate the von Mises distribution by ${ }_{463}$ a Gaussian distribution because noise is sufficiently small. 464 In this example we assume body-fixed frame azimuth and ${ }_{465}$ elevation measurement errors have standard deviations of 466 $\sigma_{\Theta}=0.5^{\circ}$ and $\sigma_{\Phi}=2^{\circ}$.

Samples of Gaussian error with these standard deviations 468 were added to body-fixed frame $\left(B_{4}\right)$ elevation and azimuth 469 measurements calculated as described in Section III. These 470 were converted to DOA measurements referenced to the INS 471 


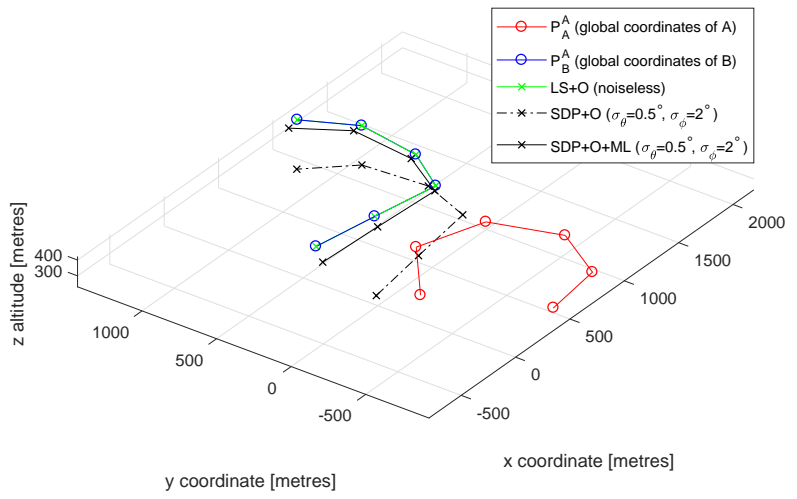

Fig. 2. Recovery of global coordinates of Agent B for recorded trajectories. Errors are $\sigma_{\theta}=0.5^{\circ}$ and $\sigma_{\phi}=2^{\circ}$ with respect to body fixed frame for the DOA measurements

472 frame $B_{3}$. The SDP+O algorithm was used to obtain $\overline{\boldsymbol{R}}$ and $\overline{\boldsymbol{t}}$ 473 using the agents' position coordinates in their respective nav474 igation frames and the noisy DOA values. The reconstructed 475 trajectory $\overline{\boldsymbol{p}_{B}^{\boldsymbol{A}_{1}}}$ is plotted in Figure 2 with the dotted black line. ${ }_{476}$ Position data of the reconstructed trajectory $\overline{p_{B}^{A_{1}}}$ are tabulated 477 in [22].

478 Remark 1. The accuracy of the $S D P+O$ solution in the 479 noiseless case was observed to deteriorate when entries in the 480 true translation vector ( $t_{i}$ for $i=1,2,3$ ) are large. This is due 481 to a form of inherent regularisation in the SDP solver Yalmip 482 [28]. When the approximate magnitude of the norm $\left\|\boldsymbol{t}_{\boldsymbol{A}_{\mathbf{1}}}^{\boldsymbol{B}_{\mathbf{2}}}\right\|$ 483 is known, one approach is to introduce a scaling coefficient 484 before entries $t_{i}$ for $i=1,2,3$ in equations (9) and (10) equal 485 to the approximate norm of $\left\|\boldsymbol{t}_{\boldsymbol{A}_{\mathbf{1}}}^{\boldsymbol{B}_{\mathbf{1}}}\right\|$.

${ }_{486}$ In [22], we discuss a controlled shifting algorithm which 487 may be applied if an approximation of $\boldsymbol{t}_{\boldsymbol{A}_{1}}^{B_{2}}$ is known a priori.

\section{MAXIMUM LiKeliHOOD ESTIMATION}

489 This section presents a maximum likelihood estimation 490 (ML) method to optimise estimates $\overline{\boldsymbol{R}}$ and $\overline{\boldsymbol{t}}$ which were 491 obtained using the SDP+O algorithm. The MLE refinement 492 uses the DOA measurements expressed with respect to the 493 body-fixed frame $B_{4}$, and the known values for $\sigma_{\Theta}$ and $\sigma_{\Phi}$ 494 describing the distribution of DOA errors. A non-linear log495 likelihood function for DOA measurement error is derived, 496 and because the minimum of the function cannot be found 497 analytically, we employ an iterative gradient descent approach.

\section{A. Likelihood Function Derivation}

499 In this section, DOA values are always expressed with 500 respect to the body-fixed frame of Agent B $\left(B_{4}\right)$ to exploit the 501 independence of azimuth and elevation measurement errors. 502 This is a change from Sections III and IV, in which DOA 503 measurements were generally expressed with respect to the 504 local INS frame $B_{2}$. The transformation between coordinate 505 frames $B_{2}$ and $B_{4}$ is known to Agent B.
Suppose body-fixed frame measurements of azimuth and 500 elevation $\Theta(k)$ and $\Phi(k)$ are contaminated by zero mean 507 Gaussian noise as follows:

- $\widetilde{\Theta}(k)=\Theta(k)+\xi_{\Theta}, \quad \xi_{\Theta} \sim N\left(0, \sigma_{\Theta}{ }^{2}\right)$

- $\widetilde{\Phi}(k)=\Phi(k)+\xi_{\Phi}, \quad \xi_{\Phi} \sim N\left(0, \sigma_{\Phi}{ }^{2}\right)$

To calculate noiseless azimuth and elevation measurements, 511 an expression must be derived for the position of Agent A in 512 Agent B's body-fixed frame $B_{4}$. Observe that

$$
\overline{\boldsymbol{p}_{\boldsymbol{A}}^{\boldsymbol{B u}^{\mathbf{4}}}}(k)=\boldsymbol{R}_{\boldsymbol{B}_{\mathbf{2}}}^{\boldsymbol{B}_{4}}(k)\left(\boldsymbol{R}_{\boldsymbol{A}_{\mathbf{1}}}^{\boldsymbol{B}_{2}} \boldsymbol{p}_{\boldsymbol{A}}^{\boldsymbol{A}_{1}}(k)+\boldsymbol{t}_{\boldsymbol{A}_{\mathbf{1}}}^{\boldsymbol{B}_{2}}\right)+\boldsymbol{t}_{\boldsymbol{B}_{\mathbf{2}}}^{\boldsymbol{B}_{4}}(k)
$$

To help distinguish coordinate reconstructions based on es- 516 timates of $\overline{\boldsymbol{R}}$ and $\overline{\boldsymbol{t}}$ from true coordinates, reconstructed ${ }_{517}$ positions will be explicitly expressed as functions of $\bar{R}$ and $\bar{t}$ : 518

$$
\overline{\boldsymbol{p}_{\boldsymbol{A}}^{\boldsymbol{B 4}}}(k, \overline{\boldsymbol{R}}, \overline{\boldsymbol{t}})=\boldsymbol{R}_{\boldsymbol{B}_{\mathbf{2}}}^{\boldsymbol{B}_{4}}(k)\left(\overline{\boldsymbol{R}} \boldsymbol{p}_{\boldsymbol{A}}^{\boldsymbol{A}_{\mathbf{1}}}(k)+\overline{\boldsymbol{t}}\right)+\boldsymbol{t}_{\boldsymbol{B}_{\mathbf{2}}}^{\boldsymbol{B}_{4}}(k)
$$

By definition of azimuth and elevation in Section II:

$$
\begin{aligned}
& \theta_{B_{4}}(k, \overline{\boldsymbol{R}}, \overline{\boldsymbol{t}})=\arcsin \left(\frac{\overline{\boldsymbol{p}_{\boldsymbol{A}}^{\boldsymbol{B 4}}}(k, \overline{\boldsymbol{R}}, \overline{\boldsymbol{t}})_{z}}{\left\|\overline{\boldsymbol{p}_{\boldsymbol{A}}^{\boldsymbol{B 4}}}(k, \overline{\boldsymbol{R}}, \overline{\boldsymbol{t}})\right\|}\right) \\
& \phi_{B_{4}}(k, \overline{\boldsymbol{R}}, \overline{\boldsymbol{t}})=\operatorname{atan} 2\left(\overline{\boldsymbol{p}_{\boldsymbol{A}}^{\boldsymbol{B 4}}}(k, \overline{\boldsymbol{R}}, \overline{\boldsymbol{t}})_{y}, \overline{\boldsymbol{p}_{\boldsymbol{A}}^{\boldsymbol{B 4}}}(k, \overline{\boldsymbol{R}}, \overline{\boldsymbol{t}})_{x}\right)
\end{aligned}
$$

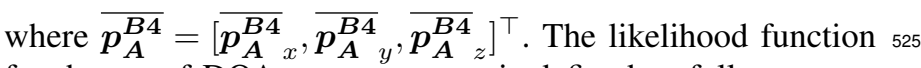
for the set of DOA measurements is defined as follows: 526

$$
\begin{aligned}
& \mathcal{L}\left(\boldsymbol{p}_{\boldsymbol{A}}^{\boldsymbol{A}_{\boldsymbol{1}}}, \boldsymbol{p}_{\boldsymbol{B}}^{\boldsymbol{B}_{\mathbf{2}}} \mid \overline{\boldsymbol{R}}, \overline{\boldsymbol{t}}\right) \\
& =\frac{1}{\sigma_{\Theta} \sqrt{2 \pi}} \prod_{k=1}^{K} \exp \left[-\frac{\left(\widetilde{\theta}_{B_{4}}(k)-\theta_{B_{4}}(k, \overline{\boldsymbol{R}}, \overline{\boldsymbol{t}})\right)^{2}}{2 \sigma_{\Theta}^{2}}\right] \\
& \times \frac{1}{\sigma_{\Phi} \sqrt{2 \pi}} \prod_{k=1}^{K} \exp \left[-\frac{\left(\widetilde{\phi}_{B_{4}}(k)-\phi_{B_{4}}(k, \overline{\boldsymbol{R}}, \overline{\boldsymbol{t}})\right)^{2}}{2 \sigma_{\Phi}^{2}}\right]
\end{aligned}
$$

It can be shown that maximising $\mathcal{L}\left(\boldsymbol{p}_{\boldsymbol{A}}^{\boldsymbol{A}_{\boldsymbol{1}}}, \boldsymbol{p}_{\boldsymbol{B}}^{\boldsymbol{B}_{2}} \mid \overline{\boldsymbol{R}}, \overline{\boldsymbol{t}}\right)$ is equiv- 531 alent to minimising

$$
\sum_{k=1}^{K}\left[\frac{\left(\widetilde{\theta}_{B_{4}}(k)-\theta_{B_{4}}(k, \overline{\boldsymbol{R}}, \overline{\boldsymbol{t}})\right)^{2}}{2 \sigma_{\Theta}^{2}}+\frac{\left(\widetilde{\phi}_{B_{4}}(k)-\phi_{B_{4}}(k, \overline{\boldsymbol{R}}, \overline{\boldsymbol{t}})\right)^{2}}{2 \sigma_{\Phi}^{2}}\right]
$$

\section{B. Optimisation using gradient descent}

534

Possible parametrisations for the rotation matrix $\overline{\boldsymbol{R}}$ include ${ }_{535}$ Euler angles, quaternion representation and Rodrigues rotation ${ }_{536}$ formula. In this paper we parametrise $\overline{\boldsymbol{R}}$ by a 3 -vector of Euler ${ }_{537}$ angles, and $\bar{t}$ is a 3 -vector. This defines a mapping from $\mathbb{R}^{6} \rightarrow 538$ $\overline{\boldsymbol{R}}, \overline{\boldsymbol{t}}$, and the gradient of (27) can be expressed as a vector in ${ }_{539}$ $\mathbb{R}^{6}$. The log-likelihood function is non-linear with respect to 540 this $\mathbb{R}^{6}$ parametrisation of $\overline{\boldsymbol{R}}$ and $\overline{\boldsymbol{t}}$. As a result, this function ${ }^{541}$ may be non-convex, meaning the equation $\mathcal{D} \log \mathcal{L}=0$ may ${ }_{542}$ have multiple solutions, with only one of these being the global ${ }_{543}$ minimum. A gradient descent algorithm is therefore initialised 544 using the result of the $\mathrm{SDP}+\mathrm{O}$ method, and is used to converge ${ }_{545}$ towards a local minimum, which it is hoped will be the global 546 minimum or close to it. In our investigations, we employed a 547 back-tracking line search algorithm discussed in [29]. External 548 solvers such as Yalmip using second-order methods may yield 549 faster convergence than a hard-coded approach.

513

.

.


${ }_{551}$ C. Example of $M L$ refinement of $S D P+O$ solution

552 In this subsection, we demonstrate the benefits of max553 imum likelihood estimation. ML was performed using the 554 real flight trajectory data presented in Section III. The re555 sulting reconstructed trajectory $\overline{p_{A}^{B_{2}}}$ is presented in Figure 5562 as the solid black line, and its coordinates are tabulated 557 in [22]. Additionally, in this section we present the decrease 558 and convergence in the value of frame rotation error and 559 reconstructed position error ${ }^{7}$ over successive iterations of the 560 gradient descent algorithm in Figures $3 \mathrm{a}$ and $3 \mathrm{~b}$.

561 The error in INS frame rotation is reduced by over $60 \%$, 562 and the reconstructed position error of Agent B is reduced 563 by over $70 \%$ by iterating the gradient descent algorithm. ${ }_{564}$ This represents a significant gain with respect to the SDP+O 565 estimate, which served as the initialisation point of the gradient 566 descent. Monte Carlo simulations covering a large set of ${ }_{567}$ trajectories are presented in Section VI.

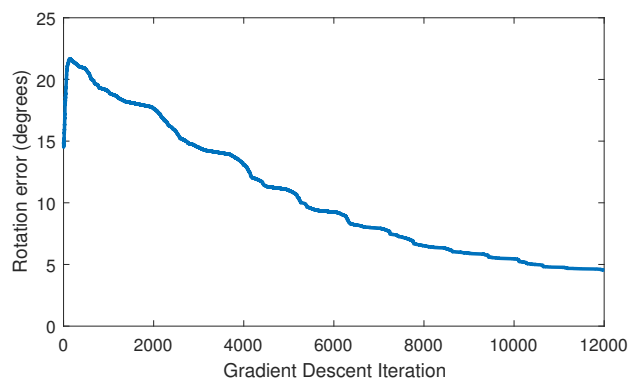

(a) Rotation error in degrees

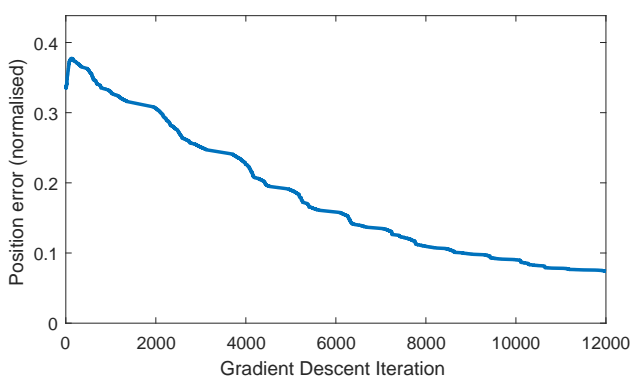

(b) Reconstructed position error

Fig. 3. Improvement in rotation and reconstructed position error using ML for real trajectory pair

568

569 571 frame azimuth and elevation measurements, and then discuss 572 trajectories which make localisation difficult.

573 In the preliminary conference paper [21] found that the ${ }_{574} \mathrm{LS}+\mathrm{O}$ method collapsed when small amounts of noise were 575 introduced to DOA measurements, whereas rotation error 576 increased linearly with respect to DOA measurement noise 577 when using the SDP+O method. The SDP+O method is the 578 superior method, and there is no reason to employ LS+O.

\footnotetext{
${ }^{7}$ Metrics are defined in the sequel, see Section VI-A below.

${ }^{8}$ These trajectories satisfy a set of assumptions detailed in [22].
}

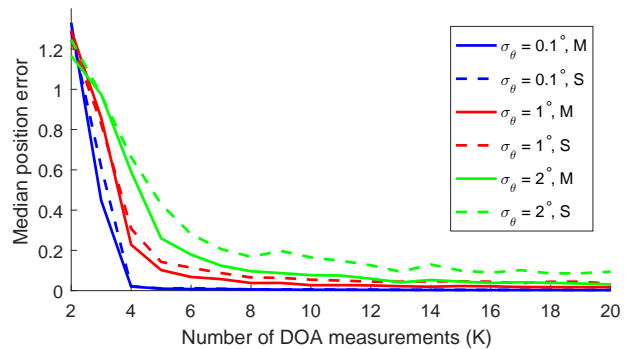

Fig. 4. Median error $\overline{\left(\boldsymbol{p}_{B}^{\boldsymbol{A}_{1}}\right.}$ ) vs. number of DOA measurements used to solve $\mathrm{SDP}+\mathrm{O}(\mathrm{S})$ and SDP+O+ML (M) from $K=2$ to $K=20$.

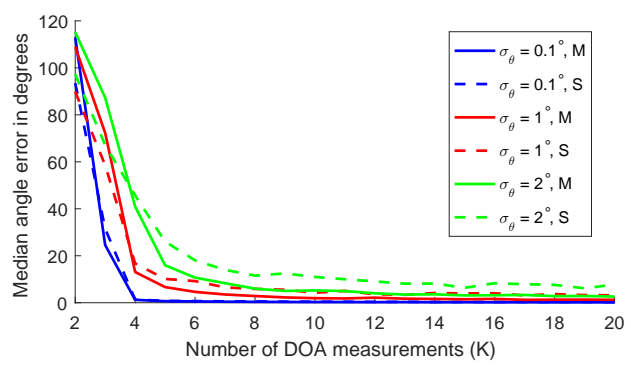

Fig. 5. Median $d\left(\overline{\boldsymbol{R}}, \boldsymbol{R}_{\boldsymbol{A}}^{\boldsymbol{B}}\right)$ vs. number of DOA measurements used to solve $\mathrm{SDP}+\mathrm{O}(\mathrm{S})$ and SDP+O+ML (M) from $K=2$ to $K=20$

\section{A. Metrics for error in $\overline{\boldsymbol{R}}$ and $\overline{\boldsymbol{t}}$}

579

This paper uses the geodesic metric for rotation [30]. All 580 sequences of rotations in three dimensions can be expressed 581 as one rotation about a single axis [31]. The geodesic metric ${ }_{582}$ on $S O(3)$ defined by

$$
d\left(\boldsymbol{R}_{\mathbf{1}}, \boldsymbol{R}_{\mathbf{2}}\right)=\arccos \left(\frac{\operatorname{tr}\left(\boldsymbol{R}_{\mathbf{1}}^{\top} \boldsymbol{R}_{\mathbf{2}}\right)-1}{2}\right)
$$

is the magnitude of angle of rotation about this axis [32]. 585 Where $\boldsymbol{R}_{\boldsymbol{A}}^{\boldsymbol{B}}$ is known, the error of rotation $\overline{\boldsymbol{R}}$ is defined as 586 $d\left(\overline{\boldsymbol{R}}, \boldsymbol{R}_{\boldsymbol{A}}^{\boldsymbol{B}}\right)$. Position error is defined as the average Euclidian 587 distance between true global coordinates of Agent B, and ${ }_{588}$ estimated global coordinates over the $K$ measurements taken, 589 divided (to secure normalisation) by the average distance 590 between aircraft.

$$
\operatorname{error}\left(\overline{\boldsymbol{p}_{\boldsymbol{B}}^{\boldsymbol{A}_{\mathbf{1}}}}\right)=\frac{\sum_{k}\left\|\overline{\boldsymbol{p}_{\boldsymbol{B}}^{\boldsymbol{A}_{\mathbf{1}}}}(k)-\boldsymbol{p}_{B}^{\boldsymbol{A}_{\mathbf{1}}}(k)\right\|}{K d}
$$

where $\overline{\boldsymbol{p}_{B}^{\boldsymbol{A}_{1}}}(k)=\overline{\boldsymbol{R}}^{\top}\left(\boldsymbol{p}_{\boldsymbol{B}}^{\boldsymbol{B}_{2}}-\overline{\boldsymbol{t}}\right)$, and $d$ represents the average 593 distance between aircraft.

\section{B. Monte Carlo Simulations using $S D P+O$ and $M L$}

595

In this subsection, we summarise the results of Monte 596 Carlo simulations to evaluate the expected performance of the ${ }_{597}$ $\mathrm{SDP}+\mathrm{O}$ method and the SDP+O+ML method.

598

Pairs of realistic trajectories for Agents A and B are 599 generated in accordance with a series of assumptions related 600 to real flight dynamics listed in the extended version of this 601 paper [22]. To represent the drift in the INS of Agent B, 602 rotations $\boldsymbol{R}_{\boldsymbol{A}_{1}}^{\boldsymbol{B}_{2}}$ were generated by independently sampling 603 three Euler angles $\alpha, \beta, \gamma$ where $\alpha, \beta, \gamma \sim U(-\pi, \pi)$, and 604 
605 translations $\boldsymbol{t}_{\boldsymbol{A}_{1}}^{\boldsymbol{B}_{\mathbf{2}}}=\left[t_{1}, t_{2}, t_{3}\right]^{\top}$ were generated by sampling 606 entries $t_{1}, t_{2}, t_{3} \sim U(-600,600)$.

${ }_{607}$ As discussed in Section IV-E, we assume the standard devi608 ations of measurement error in the body-fixed frame $B_{4}$ satisfy $609 \sigma_{\Phi}=4 \sigma_{\Theta}$. We vary the DOA error by $\sigma_{\Theta} \in\left\{0.1^{\circ}, 1^{\circ}, 2^{\circ}\right\}$. ${ }_{610}$ Errors in the order of $\sigma_{\Theta}=0.1^{\circ}$ are representative of an 611 optical sensor, whereas the larger errors are representative of 612 antenna-based (RF) measurements.

613 For each value of $\sigma_{\Theta}$ studied, and for each number of DOA 614 measurements $K$ from 2 to 20, we simulated 100 different 615 realistic UAV trajectory pairs (Agent A and Agent B). For 616 each trajectory pair, localisation was performed using the ${ }_{617} \mathrm{SDP}+\mathrm{O}$ method, and metrics $d\left(\overline{\boldsymbol{R}}, \boldsymbol{R}_{\boldsymbol{A}}^{\boldsymbol{B}}\right)$ and $\operatorname{error}\left(\overline{\boldsymbol{P}_{\boldsymbol{B}}^{\boldsymbol{A}_{\mathbf{1}}}}\right)$, 618 were calculated. The ML method was then used to enhance the 619 result of the $\mathrm{SDP}+\mathrm{O}$ method, and the error metrics were re620 calculated. After all simulations were completed, the median ${ }^{9}$ 621 values of $d\left(\overline{\boldsymbol{R}}, \boldsymbol{R}_{\boldsymbol{A}}^{\boldsymbol{B}}\right)$ and $\operatorname{error}\left(\overline{\boldsymbol{P}_{\boldsymbol{B}}^{\boldsymbol{A}_{\mathbf{1}}}}\right)$ for both the $\mathrm{SDP}+\mathrm{O}$ 622 and SDP+O+ML methods were calculated across each set 100 623 simulations. The results of the Monte Carlo simulations are ${ }_{624}$ plotted in Figures 4 and 5.

${ }_{625}$ Median $d\left(\overline{\boldsymbol{R}}, \boldsymbol{R}_{\boldsymbol{A}}^{\boldsymbol{B}}\right)$ and $\operatorname{error}\left(\overline{\boldsymbol{P}_{\boldsymbol{B}}^{\boldsymbol{A}_{\mathbf{1}}}}\right)$ errors decrease signif626 icantly when 4 or more DOA measurements $(K)$ are used. ${ }_{627}$ Both metrics show an asymptotic limit to performance across 628 all three noise levels as the number of DOA measurements $(K)$ 629 increases. Median rotation error $d\left(\overline{\boldsymbol{R}}, \boldsymbol{R}_{\boldsymbol{A}}^{\boldsymbol{B}}\right)$ and $\operatorname{error}\left(\overline{\boldsymbol{p}_{\boldsymbol{B}}^{\boldsymbol{A}_{\mathbf{1}}}}\right)$ 630 appear to exhibit similar asymptotic performance gain over the 631 number of DOA measurements $K$ up to 20 .

\section{${ }_{632}$ C. Unsuitable trajectories for localisation}

633 In this subsection we are motivated to identify trajectories ${ }_{634}$ of Agents A and B which may lead to multiple solutions for ${ }_{635} \overline{\boldsymbol{R}}$ and $\overline{\boldsymbol{t}}$ in the noiseless case, and consequently unreliable 636 solutions in the noisy case. We discuss three scenarios:

\section{1) Agent A's motion is planar}

638 2) The agents' trajectories produce equal DOA measure639 ments with respect to Agent B's INS frame.

640 3) Agent A's trajectory is a point or straight line

641 The first scenario is an example of conditional unsuitability. ${ }_{642}$ When Agent A's motion is planar, matrix $\boldsymbol{A}$ in Eqn. (11) 643 is rank deficient, and a unique solution cannot be obtained 644 by solving Eqn. (11). In contrast, by introducing quadratic 645 constraints through SDP, the correct solution is obtained.

646 The second and third scenarios are examples where the 647 inability to discern a unique solutions is not because of an 648 algorithmic deficiency, but rather because there is geometric 649 ambiguity arising from unsuitable trajectories.

650 In the second scenario, DOA measurements expressed with 651 respect to the local INS frame $B_{2}$ are equal at each time 652 instant. This is illustrated by an example in Fig. 6. A sim653 ilar problem is expected in the far field case, where the ${ }_{654}$ distance between Agents A and B is sufficiently large that 655 DOA measurements become approximately equal despite each 656 agent's trajectory remaining arbitrary. In these cases, multiple 657 solutions exist for $t_{A_{1}}^{B_{2}}$.

\footnotetext{
${ }^{9}$ For asymmetric distributions such as nonnegative errors (which may contain extreme outliers), the median is a superior measure of central tendency than the mean [33], [34].
}

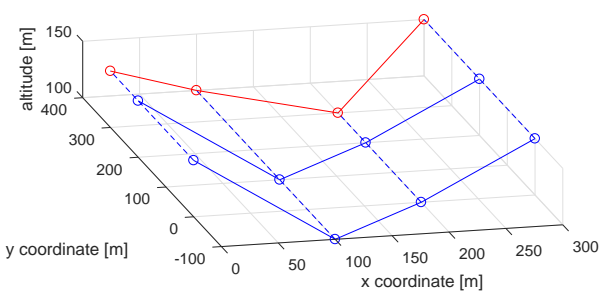

Fig. 6. Illustration of trajectory pairs leading to equal DOA measurements (disconnected blue lines) over $K$ measurement instants. SDP+O+ML algorithm unable to discern distance from which Agent B (solid blue) observes Agent A (red).

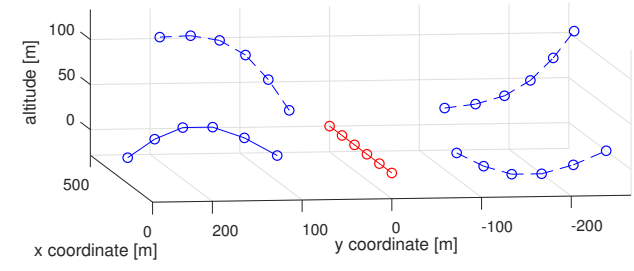

Fig. 7. Illustration of straight line motion of Agent A (red, trajectory given by $(x(t), y(t), z(t))=(100 t, 0,0))$. Agent B (blue) observes Agent A through an unaligned INS frame. In this figure, the solid blue trajectory is the actual path of Agent B. However, each dotted blue line is also an admissible solution.

In the third scenario, Agent A's trajectory appears similar 658 from multiple perspectives. As a consequence, the localisation 659 process may be incapable of determining the direction from 660 which DOA measurements were taken with respect to the 661 global frame. For example, if Agent A follows a straight 662 line, the same set of recorded DOA measurements may be ${ }_{663}$ achieved by viewing Agent A from any direction in a circle ${ }_{664}$ perpendicular to Agent A's motion and centred at Agent A's 665 trajectory. This is illustrated in Figure 7.

666

\section{THREE-AGENT EXTENSION AND BEYOND}

This section explores a novel extension to the SDP+O+ML 668 algorithm to localise two GPS-denied agents efficiently. Triv- 669 ially, each GPS-denied aircraft could measure DOA of the 670 GPS-equipped agent's broadcast of its position, and use the 671 $\mathrm{SDP}+\mathrm{O}+\mathrm{ML}$ algorithm independently of each other to estimate 672 drift in their local frames. We are motivated to determine 673 whether a trilateral $^{10}$ algorithm may be more resilient to DOA 674 measurement error and/or unsuitable trajectories, and may 675 perhaps require fewer DOA measurements from each aircraft 676 than simply repeating the two-agent localisation algorithm 677 with each GPS-denied agent. We introduce a GPS-denied 678 Agent $\mathrm{C}$, whose local INS frame has rotation and translation 679 parameters $\boldsymbol{R}_{\boldsymbol{A}_{1}}^{\boldsymbol{C}_{2}}$ and $\boldsymbol{t}_{\boldsymbol{A}_{1}}^{\boldsymbol{C}_{2}}$ with respect to the global frame. We 680 conclude this section by discussing the challenges involved in 681 generalising our findings to arbitrary $n$-agent networks.

682

\footnotetext{
${ }^{10}$ In this section we relax the condition preventing GPS-denied agents from broadcasting signals
} 
${ }_{683}$ A. Measurement process in three-agent network

684 To describe measurements within a network of more than 685 two agents, one minor notation change is required: DOA mea686 surements made by Agent I towards Agent J will henceforth 687 be expressed in the INS coordinate frame of Agent I as $\left(\theta_{I_{2}}^{J}\right.$, $\left.688 \phi_{I_{2}}^{J}\right)$. At each time instant $k$ in the discrete-time process:

689 - Agents A and B interact as per the two-agent case.

690 - Agent $\mathrm{C}$ receives the broadcast of Agent A's global 691 coordinates, and measures this signal's DOA with respect 692 to frame $C_{2}$, which we denote $\left(\theta_{C_{2}}^{A}, \phi_{C_{2}}^{A}\right)$.

693 - Agent $\mathrm{C}$ broadcasts its position with respect to its INS 694 frame $\boldsymbol{p}_{\boldsymbol{C}}^{\boldsymbol{C}_{2}}$, as well as the measurement $\left(\theta_{C_{2}}^{A}, \phi_{C_{2}}^{A}\right)$ to 695 Agent $\mathrm{B}$, who also takes a DOA measurement towards 696 Agent $\mathrm{C}$. This measurement is denoted $\left(\theta_{B_{2}}^{C}, \phi_{B_{2}}^{C}\right)$.

${ }_{697}$ All DOA and position measurements are relayed to Agent B, 698 who performs the localisation algorithm discussed below.

\section{${ }_{699}$ B. Forming system of linear equations in three-agent network}

700 In Section III, the linear system $\boldsymbol{A} \boldsymbol{\Psi}=\boldsymbol{b}$ was formed using 701 relations stemming from the collinearity of the vector $\left(\boldsymbol{p}_{\boldsymbol{A}}^{\boldsymbol{B}_{\mathbf{2}}}-\right.$ ${ }_{702} \boldsymbol{p}_{\boldsymbol{B}}^{\boldsymbol{B}_{\mathbf{2}}}$ ), and the vector in the direction of DOA measurement ${ }_{703}\left(\theta_{B_{2}}^{A}, \phi_{B_{2}}^{A}\right)$. We refer to this system of equations as $\boldsymbol{S}_{\boldsymbol{A} \boldsymbol{B}}$, 704 where the subscript references the agents involved. A similar 705 system $S_{A C}$ can be constructed independently using Agent ${ }_{706}$ C's DOA measurements towards Agent A and $\boldsymbol{p}_{\boldsymbol{C}}^{\boldsymbol{C}_{2}}$.

707 In the three-agent network, Agent B also measures the 708 DOA towards Agent C's broadcast, with respect to Agent $709 \mathrm{~B}$ 's local INS frame $B_{2}$. To exploit the collinearity of the 710 vectorial representation of the DOA measurement $\left(\theta_{B_{2}}^{C}, \phi_{B_{2}}^{C}\right)$ 711 and $\left(\boldsymbol{p}_{\boldsymbol{C}}^{\boldsymbol{B}_{\mathbf{2}}}-\boldsymbol{p}_{\boldsymbol{B}}^{\boldsymbol{B}_{2}}\right)$, an expression for the position coordinate 712 vector $\boldsymbol{p}_{\boldsymbol{C}}^{\boldsymbol{B}_{2}}$ is required. As achieved in equations (7) and (8) 713 in Section III, this position may be expressed in terms of ${ }_{714}$ entries of $\boldsymbol{R}_{\boldsymbol{C}_{2}}^{\boldsymbol{B}_{2}}$ and $\boldsymbol{t}_{\boldsymbol{C}_{2}}^{\boldsymbol{B}_{2}}$, and the linear system $\boldsymbol{S}_{\boldsymbol{B} \boldsymbol{C}}$ may 715 be defined similarly to $\boldsymbol{S}_{\boldsymbol{A} B}$ in Section III. Systems $\boldsymbol{S}_{\boldsymbol{A} \boldsymbol{B}}$, ${ }_{716} \boldsymbol{S}_{A C}$ and $\boldsymbol{S}_{B C}$ can be assembled, forming a large system of 717 linear equations $\boldsymbol{S}_{\boldsymbol{A B C}}$ with 36 scalar unknowns (9 rotation 718 matrix entries and 3 translation vector entries per agent pair). 719 At each time instant $k$ for $k=1, \ldots, K$, two linear equations 720 are obtained from each DOA measurement of $\left(\theta_{B_{2}}^{A}, \phi_{B_{2}}^{A}\right)$, ${ }_{721}\left(\theta_{C_{2}}^{A}, \phi_{C_{2}}^{A}\right)$ and $\left(\theta_{B_{2}}^{C_{2}}, \phi_{B_{2}}^{C_{2}}\right)$. As a result, 6 linear equations 722 are obtained at each time instant. Performing the measurement 723 process 6 times $(K=6)$ produces 36 linear equations. Gener724 ically, in the noiseless case, a unique solution therefore exists 725 for $K=6$ time instants. When using only the LS method, the 726 three-agent localisation problem requires the same minimum 727 number of time instants as solving two independent two-agent 728 localisation problems concurrently, yet requires more DOA 729 measurements than the sum of the number of measurements 730 required in two separate two-agent localisation problems. ${ }_{731}$ However, quadratic relationships between $R_{A_{1}}^{B_{2}}, t_{A_{1}}^{B_{2}}, R_{A_{1}}^{C_{2}}$, ${ }_{732} t_{A_{1}}^{C_{2}}, R_{B_{2}}^{C_{2}}$ and $t_{B_{2}}^{C_{2}}$ significantly reduce the required number 733 of time instants $(K)$ at which measurements occur.

\section{${ }_{734}$ C. Quadratic constraints in three-agent network and example}

735 It is possible, using the rotational and translational relation736 ships between the three frames, to obtain a total of 99 linearly

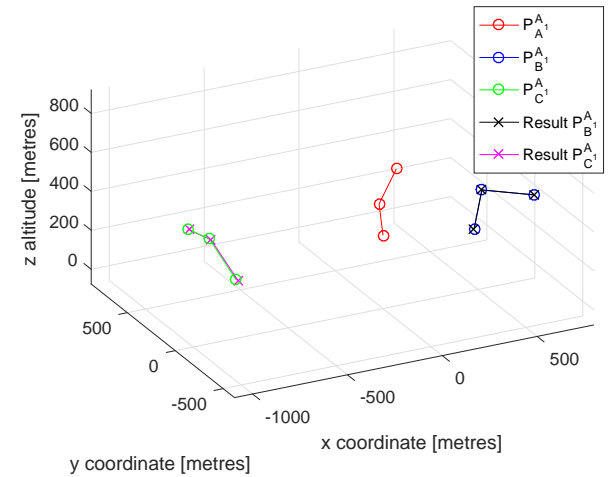

Fig. 8. Illustration of example of successful localisation within three-agent network in noiseless case for $\mathrm{K}=3$

independent quadratic constraints for a system of 36 unknown 737 variables. Exact details are given in [22] for the interested 738 reader, but omitted here for spatial considerations.

739

Rank-relaxed semidefinite programming can be used to 740 obtain solutions for each INS frame's rotation and translation 741 with respect to the global frame, and the Orthogonal Procrustes 742 algorithm can be applied to each individual resulting rotation 743 matrix. This defines the three-agent SDP+O method. $\quad 744$

To illustrate successful localisation in the three-agent case, 745 realistic trajectories were defined for Agents A, B and C 746 for $K=3$ time instants. These are presented in Figure 747 8. Only Agents B and C were assigned random INS frame 748 rotations and translations as prescribed in Section VI, and 749 the three-agent SDP+O method was used to obtain estimates 750 of $R_{A_{1}}^{B_{2}}, \boldsymbol{t}_{\boldsymbol{A}_{1}}^{B_{2}}, \boldsymbol{R}_{\boldsymbol{A}_{1}}^{C_{2}}$ and $\boldsymbol{t}_{\boldsymbol{A}_{1}}^{\boldsymbol{C}_{2}}$. Each directional measurement 751 consists of two scalar measurements, and hence a total of 752 $3 \times 2 \times K=18$ scalar measurements were obtained. Locali- 753 sation was successful, which demonstrates that only 3 time 754 instants $(K=3)$ are required for the three-agent $\mathrm{SDP}+\mathrm{O} 755$ algorithm to obtain the exact solution in the noiseless case. 756 Earlier, it was established that a minimum of 6 time instants 757 were required to achieve a unique solution in the three-agent 758 case using $\mathrm{LS}+\mathrm{O}$, and a minimum of 4 time instants were 759 required to achieve a unique solution in the two-agent case 760 using SDP+O. We have therefore demonstrated that a trilateral 761 algorithm can achieve localisation of two GPS-denied agents 762 in fewer measurement time instants than applying the bilateral 763 algorithm twice independently. We note that this extension to 764 three-agents is not applicable if the measurement graph is a 765 tree because measurements are required between each pair of 766 agents within the three-agent network.

767

\section{Challenges in extension to n-agent networks}

768

Advancing to arbitrary $n$-agent networks requires results 769 on bearing rigidity of a graph. Though results exist when 770 all agents share the same reference frame [35]-[37], there 771 is no such result when, as in our problem, agents have 772 different reference frames. We note that algebraic conditions 773 for $3 \mathrm{D}$ bearing localisability based on the rank of generalised 774 versions of the rigidity matrix have recently been identified 775 in [25] and [23]. There is also the risk of an explosion in 776 
777 computational complexity due to a potentially exponential 778 increase in the number of variables (entries of rotation matrices 779 and translation vectors) that need to be determined. Further 780 discussion can be found in [22].

\section{CONCLUSION}

782 This paper studied a cooperative localisation problem be783 tween a GPS-denied and a GPS-enabled UAV. A localisa784 tion algorithm was developed in two stages. We showed 785 that a linear system of equations built from six or more 786 measurements yielded the localisation solution for generic 787 trajectories. The second stage considered the inclusion of 788 quadratic constraints due to rotation matrix constraints. Rank 789 relaxed semidefinite programming was used, and the solution 790 adjusted using the Orthogonal Procrustes algorithm. This gave 791 the algorithm greater resilience to noisy measurements and 792 unsuitable trajectories. Maximum likelihood estimation was 793 then used to improve the algorithm's results. Simulations were 794 presented to illustrate the algorithm's performance. Finally, 795 an approach was outlined to extend the two-agent solution 796 to a three agent network in which only one agent has global 797 localisation capacity. Future work may include implementation 798 on aircraft to perform localisation in real time and validate 799 our Monte Carlo analysis on measurement noise. We also 800 hope to extend our trilateral algorithm to larger networks by 801 establishing further theory on bearing rigidity when agents do 802 not share a common reference frame.

\section{REFERENCES}

[1] G. Balamurugan, J. Valarmathi, and V. Naidu, "Survey on UAV Navigation in GPS Denied Environments," in Signal Processing, Communication, Power and Embedded System (SCOPES), 2016 International Conference on. IEEE, 2016, pp. 198-204.

2] H. Bai and R. W. Beard, "Relative Heading Estimation for Target Handoff in GPS-Denied Environments," in American Control Conference (ACC), 2016. IEEE, 2016, pp. 336-341.

3] J. Morales, P. Roysdon, and Z. Kassas, "Signals of Opportunity Aided Inertial Navigation," in Proceedings of ION GNSS Conference, 2016, pp. 1492-1501.

[4] J. Morales and Z. M. Kassas, "Information fusion strategies for collaborative radio slam," in 2018 IEEE/ION Position, Location and Navigation Symposium (PLANS), April 2018, pp. 1445-1454.

5] J. Morales and Z. Kassas, "A Low Communication Rate Distributed Inertial Navigation Architecture with Cellular Signal Aiding," in 2018 IEEE 87th Vehicular Technology Conference (VTC Spring). IEEE, 2018, pp. 1-6.

[6] - ,Distributed Signals of Opportunity Aided Inertial Navigation with Intermittent Communication," in 2017 ION GNSS+ Conference, 2017, pp. 2519-2530.

[7] A. N. Bishop, B. Fidan, B. D. O. Anderson, K. Dogancay, and P. N. Pathirana, "Optimality Analysis of Sensor-Target Geometries in Passive Localization: Part 1 - Bearing-Only Localization," in Intelligent Sensors, Sensor Networks and Information, Dec 2007, pp. 7-12.

8] L. G. Taff, "Target Localization From Bearings-Only Observations," IEEE Transactions on Aerospace and Electronic Systems, vol. 33, no. 1, pp. 2-10, Jan 1997.

9] O. Tekdas and V. Isler, "Sensor Placement for Triangulation-Based Localization," IEEE Transactions on Automation Science and Engineering, vol. 7, no. 3, pp. 681-685, July 2010.

10] P. Batista, C. Silvestre, and P. Oliveira, "Navigation systems based on multiple bearing measurements," IEEE Transactions on Aerospace and Electronic Systems, vol. 51, no. 4, pp. 2887-2899, Oct 2015.

1] K. Dogancay, "Self-Localization from Landmark Bearings using Pseudolinear Estimation Techniques," IEEE Transactions on Aerospace and Electronic Systems, vol. 50, no. 3, pp. 2361-2368, July 2014.
[12] Y. Duan, R. Ding, and H. Liu, "A Probabilistic Method of Bearing-only 840 Localization by Using Omnidirectional Vision Signal Processing," in 841 Intelligent Information Hiding and Multimedia Signal Processing (IIH- 842 MSP), 2012 Eighth International Conference on, July 2012, pp. 285- 843 288.

[13] P. Batista, C. Silvestre, and P. Oliveira, "Globally Exponentially Stable 845 Filters for Source Localization and Navigation Aided by Direction 846 Measurements,' Systems \& Control Letters, vol. 62, no. 11, pp. 1065 - 847 1072, 2013.

[14] H. Bayram, J. V. Hook, and V. Isler, "Gathering Bearing Data for Target 849 Localization,' IEEE Robotics and Automation Letters, vol. 1, no. 1, pp. 850 369-374, Jan 2016.

[15] M. Gavish and A. J. Weiss, "Performance Analysis of Bearing-Only 852 Target Location Algorithms," IEEE Transactions on Aerospace and 853 Electronic Systems, vol. 28, no. 3, pp. 817-828, Jul 1992.

[16] S. Sohn, B. Lee, J. Kim, and C. Kee, "Vision-Based Real-Time Target 855 Localization for Single-Antenna GPS-Guided UAV," IEEE Transactions 856 on Aerospace and Electronic Systems, vol. 44, no. 4, pp. 1391-1401, 857 Oct 2008

[17] J. Reis, P. Batista, P. Oliveira, and C. Silvestre, "Source Localization 859 Based on Acoustic Single Direction Measurements," IEEE Transactions 860 on Aerospace and Electronic Systems, pp. 1-1, 2018.

[18] J. J. Koenderink and A. J. van Doorn, "Affine Structure From Motion," 862 J. Opt. Soc. Am. A, vol. 8, no. 2, pp. 377-385, Feb 1991.

[19] M. Ye, B. D. O. Anderson, and C. Yu, "Bearing-Only Measurement 864 Self-Localization, Velocity Consensus and Formation Control," IEEE 865 Transactions on Aerospace and Electronic Systems, vol. 53, no. 2, pp. 866 575-586, April 2017.

[20] L. Zhang, M. Ye, B. D. O. Anderson, P. Sarunic, and H. Hmam, 868 "Cooperative localisation of uavs in a gps-denied environment using 869 bearing measurements," in 2016 IEEE 55th Conference on Decision and 870 Control (CDC), Dec 2016, pp. 4320-4326.

[21] J. S. Russell, M. Ye, B. D. Anderson, H. Hmam, and P. Sarunic, 872 "Cooperative Localisation of a GPS-Denied UAV in 3-Dimensional 873 Space Using Direction of Arrival Measurements," IFAC-PapersOnLine, 874 vol. 50, no. 1, pp. 8019 - 8024, 2017, 20th IFAC World Congress. 875

[22] J. S. Russell, M. Ye, B. D. O. Anderson, H. Hmam, and 876 P. Sarunic, "Cooperative Localisation of GPS-Denied UAVs using 877 Direction of Arrival Measurements," 2018. [Online]. Available: 878 http://arxiv.org/abs/1804.04317

[23] M. Pasquetti, G. Michieletto, S. Zhao, D. Zelazo, and A. Cenedese, "A 880 Unified Dissertation on Bearing Rigidity Theory," CoRR, 2019. 881

[24] M. Arie-Nachimson, S. Z. Kovalsky, I. Kemelmacher-Shlizerman, 882 A. Singer, and R. Basri, "Global Motion Estimation from Point 883 Matches," in Proceedings of the 2012 Second International Conference 884 on 3D Imaging, Modeling, Processing, Visualization \& Transmission, 885 2012, pp. 81-88.

[25] F. Schiano and R. Tron, "The Dynamic Bearing Observability Matrix 887 Nonlinear Observability and Estimation for Multi-Agent Systems," in 888 ICRA 2018 - IEEE International Conference on Robotics and Automa- 889 tion. Brisbane, Australia: IEEE, May 2018, pp. 1-8. 890

[26] D. Eggert, A. Lorusso, and R. Fisher, "Estimating 3-D Rigid Body 891 Transformations: A Comparison of Four Major Algorithms," Machine 892 Vision and Applications, vol. 9, no. 5, pp. 272-290, Mar 1997.

[27] C. Forbes, M. Evans, N. Hastings, and B. Peacock, Statistical Distribu- 894 tions. John Wiley \& Sons, 2011.

[28] J. Löfberg, "YALMIP : A Toolbox for Modeling and Optimization 89 in MATLAB," in In Proceedings of the CACSD Conference, Taipei, 897 Taiwan, 2004

[29] P. S. Stanimirović and M. B. Miladinović, "Accelerated Gradient Descent 899 Methods with Line Search," Numerical Algorithms, vol. 54, no. 4, pp. 900 503-520, 2010

[30] D. Q. Huynh, "Metrics for 3D Rotations: Comparison and Analysis," 902 Journal of Mathematical Imaging and Vision, vol. 35, no. 2, pp. 155- 903 164, 2009.

[31] B. Palais, R. Palais, and S. Rodi, "A Disorienting Look at Euler's 905 Theorem on the Axis of a Rotation," American Mathematical Monthly, 906 vol. 116, no. 10 , pp. 892-909, 2009.

[32] R Tron, J. Thomas, G. Loianno, K. Daniilidis, and V. Kumar, “A Distributed Optimization Framework for Localization and Formation 909 Control: Applications to Vision-Based Measurements," IEEE Control 910 Systems, vol. 36, no. 4, pp. 22-44, Aug 2016.

[33] S. Boslaugh, Statistics in a Nutshell: A Desktop Quick Reference. 912 O’Reilly Media, Inc., 2012.

[34] H. L. Harter, "The Method of Least Squares and Some Alternatives: 914 Part II," International Statistical Review, vol. 42, no. 3, pp. 235-282, 915 1974. 
917 [35] S. Zhao and D. Zelazo, "Bearing Rigidity and Almost Global Bearing918 Only Formation Stabilization," IEEE Transactions on Automatic Control, 919 vol. 61, no. 5, pp. 1255-1268, May 2016.

920 [36] D. Zelazo, A. Franchi, and P. R. Giordano, "Rigidity theory in se(2) for 921 unscaled relative position estimation using only bearing measurements," 9222014 European Control Conference (ECC), pp. 2703-2708, 2014.

923 [37] S. Zhao and D. Zelazo, "Localizability and Distributed Protocols for 924 Bearing-Based Network Localization in Arbitrary Dimensions," Auto925 matica, vol. 69, pp. $334-341,2016$. 